\title{
Experimental investigation on supercritical water gasification of organic-rich shale with low maturity for syngas production
}

\author{
Xinping Liang ${ }^{\dagger, \#}$, Qiuyang Zhao ${ }^{\ddagger, \#}$, Yu Dong ${ }^{\ddagger}$, Liejin Guo ${ }^{\ddagger, *}$, Zhijun Jin ${ }^{\dagger, *}$, and Quanyou Liu ${ }^{\dagger}$
}

\footnotetext{
† State Key Laboratory of Shale Oil and Gas Enrichment Mechanisms and Effective Development, SINOPEC Exploration and Production Research Institute, Beijing, 100083, China

‡ State Key Laboratory of Multiphase Flow in Power Engineering, Xi'an Jiaotong University, Xi'an, 710049, China

*Correspondence: lj-guo@xjtu.edu.cn; jinzj.syky@sinopec.com.
}

\section{Contents}

Table S1. Detailed experimental data of SCWG of shale. 


\begin{tabular}{|c|c|c|c|c|c|c|c|c|c|c|c|c|c|c|c|c|c|c|}
\hline No. & \begin{tabular}{|c} 
Designed \\
Temperature \\
${ }^{\circ} \mathrm{C}$
\end{tabular} & \begin{tabular}{|c|} 
Measured \\
Temperatur \\
$\mathbf{e}$
\end{tabular} & $\begin{array}{c}\text { Designed } \\
\text { Pressure } \\
\mathrm{MPa}\end{array}$ & $\begin{array}{c}\text { Measured } \\
\text { Pressure } \\
\mathrm{MPa}\end{array}$ & $\begin{array}{c}\text { Reaction } \\
\text { Time/h }\end{array}$ & $\begin{array}{c}\text { Particle Size } \\
\text { Range/ } \\
\text { mesh }\end{array}$ & \begin{tabular}{|c|} 
Designed \\
Water-shale \\
Mass Ratio
\end{tabular} & \begin{tabular}{|c|} 
Measured \\
Water-shale \\
Mass Ratio
\end{tabular} & $\begin{array}{c}\text { Shale } \\
\text { Mass } \\
\text { q }\end{array}$ & $\begin{array}{l}\text { Water } \\
\text { Mass } \\
\text { q }\end{array}$ & $\begin{array}{c}\text { Total Gas } \\
\text { Yield } \\
\mathrm{ml} / \mathrm{q}\end{array}$ & $\begin{array}{c}\mathrm{H}_{2} \\
\mathrm{~mL} \cdot \mathrm{g}^{-1}\end{array}$ & $\begin{array}{c}\mathrm{CO} \\
\mathrm{mL} \cdot \mathrm{g}^{-1}\end{array}$ & $\mid \begin{array}{c}\mathrm{CH}_{4} \\
\mathrm{~mL} \cdot \mathrm{g}^{-1}\end{array}$ & $\begin{array}{c}\mathrm{CO}_{2} \\
\mathrm{~mL} \cdot \mathrm{g}^{-1}\end{array}$ & $\begin{array}{c}\mathrm{C}_{2+} \\
\mathrm{mL} \cdot \mathrm{g}^{-1}\end{array}$ & \begin{tabular}{|c|} 
Carbon \\
Gasification \\
Efficiency
\end{tabular} & \begin{tabular}{|c|} 
Residue \\
Mass \\
q
\end{tabular} \\
\hline 1 & 500 & 502.4 & 24 & \begin{tabular}{|l|}
24.3 \\
\end{tabular} & 1 & $>150$ & \begin{tabular}{|l|l|}
5 \\
\end{tabular} & \begin{tabular}{|l|l}
5.06 \\
\end{tabular} & \begin{tabular}{|l|}
2.65 \\
\end{tabular} & 13.41 & 56.47 & 6.11 & 0.08 & 6.83 & 38.12 & 5.33 & & \begin{tabular}{|c|}
2.28 \\
\end{tabular} \\
\hline 2 & 500 & 495.7 & 24 & 24.1 & 1 & $>150$ & 5 & 5.04 & 2.66 & 13.42 & 56.01 & 5.91 & 0.10 & 6.41 & \begin{tabular}{|l|}
38.48 \\
\end{tabular} & 5.11 & 27. & 2.30 \\
\hline 3 & 550 & 547.1 & 24 & 23.9 & 1 & $>150$ & 5 & 5.08 & 2.35 & 11.95 & 66.30 & 10.31 & 0.19 & 12.00 & 38.80 & 5.00 & & 2.12 \\
\hline 4 & 550 & 551.5 & 24 & 24.4 & 1 & $>150$ & 5 & 5.09 & 2.35 & 11.95 & 65.83 & 10.77 & 0.16 & 12.56 & 38.14 & 4.20 & $28.55 \%$ & 1.93 \\
\hline 5 & 600 & 595.2 & 24 & 24.2 & 1 & $>150$ & 5 & 5.02 & 2.39 & \begin{tabular}{|l|}
11.99 \\
\end{tabular} & 91.56 & 20.52 & 0.31 & 15.03 & 52.34 & 3.36 & & 2.00 \\
\hline 6 & 600 & 601.4 & 24 & 24.1 & 1 & $>150$ & 5 & 5.04 & 2.37 & 11.98 & 87.61 & 19.94 & 0.26 & 13.57 & 50.92 & 2.92 & $34.24 \%$ & 1.99 \\
\hline 7 & 650 & 646.9 & 24 & 24.4 & 1 & $>150$ & 5 & 5.03 & 2.25 & 11.30 & 128.10 & 39.28 & 0.65 & 18.80 & 67.83 & 1.54 & & 1.77 \\
\hline 8 & 650 & 651.6 & 24 & 24.1 & 1 & $>150$ & 5 & 5.05 & 2.20 & 11.10 & 133.08 & 39.48 & 0.63 & 20.90 & 70.69 & 1.38 & $43.16 \%$ & 1.72 \\
\hline 9 & 700 & 701.6 & 24 & 23.9 & 1 & $>150$ & 5 & 5.03 & 2.11 & 10.62 & 158.96 & 56.07 & 1.17 & 21.08 & 80.64 & 0.00 & & 1.63 \\
\hline 10 & 700 & 697.2 & 24 & 23.8 & 1 & $>150$ & 5 & 5.07 & 2.10 & 10.62 & 163.18 & 54.01 & 1.09 & 23.02 & 85.06 & 0.00 & $50.0 \% \%$ & 1.48 \\
\hline 11 & 600 & 602.3 & 22 & 21.9 & 1 & $>150$ & 5 & 5.01 & 2.18 & 10.95 & 91.32 & 19.72 & 0.32 & 12.64 & 55.78 & 2.86 & $3477 \%$ & 1.85 \\
\hline 12 & 600 & 598.1 & 22 & 22.0 & 1 & $>150$ & 5 & 5.04 & 2.19 & \begin{tabular}{|l|}
11.03 \\
\end{tabular} & 89.53 & 19.40 & 0.29 & 13.80 & 53.40 & 2.64 & $34.7 / \%$ & 1.86 \\
\hline 13 & 600 & 602.7 & 23 & 23.3 & 1 & $>150$ & 5 & 5.05 & 2.27 & 11.45 & 88.52 & 18.79 & 0.36 & 14.34 & 52.40 & 2.63 & $3483 \%$ & 1.90 \\
\hline 14 & 600 & 600.8 & 23 & 23.1 & 1 & $>150$ & 5 & 5.05 & 2.27 & 11.46 & 91.39 & 19.53 & 0.27 & 13.58 & 54.76 & 3.25 & $34.83 \%$ & 1.90 \\
\hline 15 & 600 & 595.2 & 24 & 24.2 & 1 & $>150$ & 5 & 5.02 & 2.39 & 11.99 & 91.56 & 20.52 & 0.31 & 15.03 & 52.34 & 3.36 & & 2.00 \\
\hline 16 & 600 & 601.4 & 24 & 24.1 & 1 & $>150$ & 5 & 5.04 & 2.37 & 11.98 & 87.61 & 19.94 & 0.26 & 13.57 & 50.92 & 2.92 & $34.24 \%$ & 1.99 \\
\hline 17 & 600 & 602.3 & 26 & 26.2 & 1 & $>150$ & 5 & 5.04 & 2.41 & 12.13 & 89.24 & 20.04 & 0.25 & 16.03 & 49.46 & 3.46 & & 2.05 \\
\hline 18 & 600 & 603.5 & 26 & 25.9 & 1 & $>150$ & 5 & 5.05 & 2.40 & 12.11 & 87.80 & 19.04 & 0.27 & 13.85 & 51.56 & 3.08 & $44.73 \%$ & 2.05 \\
\hline 19 & 600 & 600.4 & 28 & 28.1 & 1 & $>150$ & 5 & 5.07 & 2.51 & 12.70 & 87.20 & 18.77 & 0.31 & 15.46 & 49.27 & 3.39 & & 2.16 \\
\hline 20 & 600 & 597.6 & 28 & 28.0 & 1 & $>150$ & 5 & 5.06 & 2.51 & 12.71 & 87.47 & 19.05 & 0.34 & 14.48 & 49.99 & 3.61 & $33.98 \%$ & 2.16 \\
\hline 21 & 700 & 700.1 & 24 & 24.1 & 0 & $>150$ & 5 & 5.07 & 2.11 & 10.72 & 135.45 & 46.71 & 0.88 & 18.30 & 69.14 & 0.42 & & 1.73 \\
\hline 22 & 700 & 698.4 & 24 & 24.0 & 0 & $>150$ & 5 & 5.08 & 2.11 & 10.72 & 136.77 & 42.67 & 1.12 & 20.92 & 71.40 & 0.66 & $43.45 \%$ & 1.68 \\
\hline 23 & 700 & 701.6 & 24 & 23.9 & 1 & $>150$ & 5 & 5.03 & 2.11 & 10.62 & 158.96 & 56.07 & 1.17 & 21.08 & 80.64 & 0.00 & & 1.63 \\
\hline 24 & 700 & 697.2 & 24 & 23.8 & 1 & $>150$ & 5 & 5.07 & 2.10 & 10.62 & 163.18 & 54.01 & 1.09 & 23.02 & 85.06 & 0.00 & $50.0 / \%$ & 1.48 \\
\hline 25 & 700 & 697.1 & 24 & 24.1 & 4 & $>150$ & 5 & 5.01 & 2.10 & 10.55 & 234.74 & 94.26 & 1.96 & 27.59 & \begin{tabular}{|l}
110.93 \\
\end{tabular} & 0.00 & $6602 \%$ & 1.51 \\
\hline 26 & 700 & 700.5 & 24 & 24.3 & 4 & $>150$ & 5 & 5.12 & 2.08 & \begin{tabular}{|l}
10.62 \\
\end{tabular} & 235.41 & 96.32 & 1.83 & 24.47 & \begin{tabular}{|l|l}
112.79 \\
\end{tabular} & 0.00 & $60.02 \%$ & 1.46 \\
\hline 27 & 700 & 700.8 & 24 & 24.2 & 8 & $>150$ & 5 & 5.06 & 2.10 & 10.61 & 259.50 & 107.66 & 2.11 & 27.98 & 121.75 & 0.00 & & 1.45 \\
\hline 28 & 700 & 702.1 & 24 & 24.1 & 8 & $>150$ & 5 & 5.07 & 2.08 & 10.57 & 255.26 & 105.08 & 1.95 & 30.14 & $\begin{array}{l}118.09 \\
\end{array}$ & 0.00 & $1.32 \%$ & 1.40 \\
\hline 29 & 700 & 700.3 & 24 & 24.3 & 12 & $>150$ & 5 & 5.01 & 2.11 & 10.57 & 262.37 & \begin{tabular}{|c|}
106.36 \\
\end{tabular} & 2.41 & 30.81 & \begin{tabular}{|l}
122.79 \\
\end{tabular} & 0.00 & & 1.52 \\
\hline 30 & 700 & 700.4 & 24 & 24.3 & 12 & $>150$ & 5 & 5.06 & 2.11 & \begin{tabular}{|l|l}
10.67 \\
\end{tabular} & 253.81 & \begin{tabular}{|c|c|}
107.64 \\
\end{tabular} & 2.51 & 27.89 & 115.77 & 0.00 & $1.37 \%$ & 1.71 \\
\hline 31 & 700 & 695.3 & 24 & 24.4 & 1 & $>150$ & 1 & 1.00 & 10.08 & 10.09 & 114.18 & 27.74 & 1.30 & 23.39 & \begin{tabular}{|l}
61.75 \\
\end{tabular} & 0.00 & & 8.45 \\
\hline 32 & 700 & 699.2 & 24 & 24.3 & 1 & $>150$ & 1 & 1.00 & 10.01 & 10.00 & 119.10 & 29.06 & 1.04 & 25.45 & 63.55 & 0.00 & $8 \%$ & 8.66 \\
\hline 33 & 700 & 695.8 & 24 & 24.3 & 1 & $>150$ & 2.5 & 2.53 & 4.16 & 10.54 & 130.27 & 38.80 & 0.87 & 21.38 & 69.22 & 0.00 & & 3.56 \\
\hline 34 & 700 & 702.4 & 24 & 24.1 & 1 & $>150$ & 2.5 & 2.54 & 4.11 & 10.42 & 135.51 & 39.14 & 1.13 & 23.60 & 71.64 & 0.00 & $44.36 \%$ & 3.43 \\
\hline 35 & 700 & 701.6 & 24 & 23.9 & 1 & $>150$ & 5 & 5.03 & 2.11 & 10.62 & 158.96 & 56.07 & 1.17 & 21.08 & 80.64 & 0.00 & & 1.63 \\
\hline 36 & 700 & 697.2 & 24 & 23.8 & 1 & $>150$ & 5 & 5.07 & 2.10 & 10.62 & 163.18 & 54.01 & 1.09 & 23.02 & 85.06 & 0.00 & $50.07 \%$ & 1.48 \\
\hline 37 & 700 & 700.5 & 24 & 23.6 & 1 & $>150$ & 10 & 9.97 & 1.06 & 10.58 & 158.62 & \begin{tabular}{|l|}
55.16 \\
\end{tabular} & 0.59 & 20.79 & 82.08 & 0.00 & & 0.86 \\
\hline 38 & 700 & 702.3 & 24 & 23. & 1 & $>$ & 10 & 9.99 & 1.06 & 10.61 & 163.35 & 55.44 & 0.42 & 22.75 & 84.74 & 0.00 & $49.92 \%$ & 0.81 \\
\hline 39 & 700 & 695.8 & 24 & 24.4 & 1 & $5-10$ & 5 & 5.02 & 2.12 & 10.63 & 138.13 & 53.97 & 0.72 & 19.38 & 64.06 & 0.00 & 4082 & 1.75 \\
\hline 40 & 700 & 700.1 & 24 & 24 & 1 & $5-10$ & 5 & 5.04 & 2.10 & 10.58 & 145.38 & 56.71 & 0.87 & 21.52 & 66.28 & 0.00 & $40.82 \%$ & 1.58 \\
\hline 41 & 700 & 700.9 & 24 & 24.3 & 1 & $10-20$ & 5 & 5.06 & 2.10 & 10.62 & 158.33 & 58.51 & 0.93 & 21.75 & 77.14 & 0.00 & & 1.73 \\
\hline 42 & 700 & 695.4 & 24 & 24.3 & 1 & $10-20$ & 5 & 5.00 & 2.12 & 10.60 & 169.47 & 62.49 & 1.25 & 25.03 & 80.70 & 0.00 & $8.84 \%$ & 1.66 \\
\hline 43 & 700 & 700.3 & 24 & 23.9 & 1 & $40-50$ & 5 & 5.05 & 2.10 & 10.63 & 170.47 & $\begin{array}{l}59.24 \\
\end{array}$ & 1.32 & 24.88 & 85.03 & 0.00 & & 1.68 \\
\hline 44 & 700 & 702.7 & 24 & 23.5 & 1 & $40-50$ & 5 & 5.07 & 2.09 & 10.60 & 160.77 & 56.90 & 1.12 & 21.34 & 81.41 & 0.00 & $0.80 \%$ & 1.64 \\
\hline 45 & 700 & 704.1 & 24 & 23.5 & 1 & $70-90$ & 5 & 4.99 & 2.11 & 10.54 & 154.94 & 54.27 & 1.06 & 21.14 & 78.47 & 0.00 & & 1.61 \\
\hline 46 & 700 & 700.8 & 24 & 24.2 & 1 & $70-90$ & 5 & 5.05 & 2.10 & 10.61 & 163.82 & \begin{tabular}{|l}
56.59 \\
\end{tabular} & 1.24 & 23.72 & \begin{tabular}{|l}
82.27 \\
\end{tabular} & 0.00 & $9.08 \%$ & 1.62 \\
\hline 47 & 700 & 701.6 & 24 & 23.9 & 1 & $>150$ & 5 & 5.03 & 2.11 & \begin{tabular}{|c|c|}
10.62 \\
\end{tabular} & 158.96 & \begin{tabular}{|l|}
56.07 \\
\end{tabular} & 1.17 & 21.08 & 80.64 & 0.00 & & 1.63 \\
\hline 48 & 700 & 697.2 & 24 & 23.8 & 1 & $>150$ & 5 & 5.07 & 2.10 & 10.62 & 163.18 & 54.01 & 1.09 & 23.02 & 85.06 & 0.00 & $0.07 \%$ & 1.48 \\
\hline
\end{tabular}

